\title{
Continuum-wise expansive diffeomorphisms and conservative systems
}

\section{Manseob Lee*}

\section{*Correspondence:}

Imsds@mokwon.ac.kr

Department of Mathematics,

Mokwon University, Daejeon,

302-729, Korea

\section{Springer}

\begin{abstract}
We prove that $C^{1}$-generically, continuum-wise expansive diffeomorphisms satisfy both Axiom A and the no-cycle condition. Moreover, (i) if a volume-preserving diffeomorphism belongs to the $C^{1}$-interior of the set of all continuum-wise expansive volume-preserving diffeomorphisms then it is Anosov, and (ii) $C^{1}$-generically, every continuum-wise expansive volume-preserving diffeomorphism is transitive Anosov.
\end{abstract} MSC: $37 C 20 ; 37 D 20$

Keywords: Axiom A; expansive; continuum-wise expansive; Anosov; transitive; generic property

\section{Introduction}

Let $\operatorname{Diff}(M)$ be the space of diffeomorphisms of closed $C^{\infty}$-manifolds $M$ endowed with the $C^{1}$-topology, and let $d$ denote the distance on $M$ induced from a Riemannian metric $\|\cdot\|$ on the tangent bundle $T M$. In dynamical systems, expansivity is a useful notion to study of the stability. Roughly speaking, if two points stay near for future and past iterates, then they must be equal. We say that $f$ is expansive if there is $e>0$ such that for any pair of distinct points $x, y \in M, d\left(f^{n}(x), f^{n}(y)\right)>e$ for some $n \in \mathbb{Z}$. The number $e>0$ is called an expansive constant for $f$.

For a point $x \in M$, we say that $x$ is a non-wandering point if for any neighborhood $U$ of $x$, there is $n \in \mathbb{Z}$ such that $f^{n}(U) \cap U \neq \emptyset$. Denote by $\Omega(f)$ the set of all non-wandering points of $f$. It is clear $\overline{P(f)} \subset \Omega(f)$, where $P(f)$ is the set of the periodic points of $f$, and $\overline{P(f)}$ is the closure of $P(f)$. We say that $f$ satisfies Axiom $\mathrm{A}$ if $\Omega(f)=\overline{P(f)}$ is hyperbolic. We say that $f$ is quasi-Anosov if for any $v \in T M(v \neq 0)$ the set $\left\{\left\|D f^{n}(v)\right\|: n \in \mathbb{Z}\right\}$ is unbounded. It follows that $f$ satisfies Axiom A.

For expansivity, in [1], Mañé showed that a diffeomorphism belongs to the $C^{1}$-interior of the set of all expansive diffeomorphisms if and only if $f$ is quasi-Anosov.

In this paper, we study the notion of continuum-wise expansivity which was introduced by Kato in [2]. Let $\Lambda$ be a closed set of $M$. A set $\Lambda$ is nondegenerate if the set $\Lambda$ is not reduced to one point. We say that $\Lambda \subset M$ is a subcontinuum if it is a compact connected nondegenerate subset $\Lambda$ of $M$. A diffeomorphism $f$ on $M$ is said to be continuum-wise expansive if there is a constant $e>0$ such that for any nondegenerate subcontinuum $A$ there is an integer $n=n(A)$ such that $\operatorname{diam} f^{n}(A) \geq e$, where $\operatorname{diam} S=\sup \{d(x, y): x, y \in S\}$ for any subset $S$ of $M$. Such a constant $\alpha$ is called a continuum-wise expansive constant for $f$. Note that every expansive homeomorphism is continuum-wise expansive diffeomorphism, but

C2014 Lee; licensee Springer. This is an Open Access article distributed under the terms of the Creative Commons Attribution License (http://creativecommons.org/licenses/by/2.0), which permits unrestricted use, distribution, and reproduction in any medium, provided the original work is properly cited. 
its converse is not true (see [3, Example 3.5]). For diffeomorphisms, we introduce an example. It is well known that $\mathbf{S}^{2}$ does not admit an expansive diffeomorphism, but it admits a continuum-wise expansive diffeomorphisms (see [4]).

\section{Continuum-wise diffeomorphisms}

Let $M$ be as before, and let $f \in \operatorname{Diff}(M)$. Denote by $\mathcal{E}(M)$ and $\mathcal{C W E}(M)$ the set of all expansive diffeomorphisms and the set of all continuum-wise expansive diffeomorphisms, respectively. Sakai [5] proved that $f \in \mathcal{C} \mathcal{W E}(M)$ if and only if the diffeomorphism is quasiAnosov. By Mañé's result [1], we know the following.

Theorem 2.1 The $C^{1}$-interior of $\mathcal{C W E}(M)$ coincides with the $C^{1}$-interior of $\mathcal{E}(M)$.

We say that $\Lambda$ is transitive set if there is a point $x \in \Lambda$ such that $\omega_{f}(x)=\Lambda$, where $\omega_{f}(x)$ is the $\omega$-limit set of $x$. Let $\Lambda \subset M$ be an $f$-invariant closed set. We say that $\Lambda$ admits a dominated splitting if the tangent bundle $T_{\Lambda} M$ has a continuous $D f$-invariant splitting $E \oplus F$ and there exist constants $C>0$ and $0<\lambda<1$ such that

$$
\left\|\left.D_{x} f^{n}\right|_{E(x)}\right\| \cdot\left\|\left.D_{x} f^{-n}\right|_{F\left(f^{n}(x)\right)}\right\| \leq C \lambda^{n}
$$

for all $x \in \Lambda$ and $n \geq 0$. Recently, Lee [6] showed that if a transitive set $\Lambda$ is $C^{1}$-stably continuum-wise expansive then it admits a dominated splitting.

A subset $\mathcal{R} \subset \operatorname{Diff}(M)$ is called residual if it contains a countable intersection of open and dense subsets of $\operatorname{Diff}(M)$. A dynamic property is called $C^{1}$-generic if it holds in a residual subset of $\operatorname{Diff}(M)$. We use the terminology for $C^{1}$-generic $f$ to express there is a residual subset $\mathcal{R} \subset \operatorname{Diff}(M)$, and $f \in \mathcal{R}$.

Recently, in [7], Arbieto proved that for $C^{1}$-generic $f \in \operatorname{Diff}(M), f$ is expansive then $f$ is $\Omega$-stable, that is, obeys Axiom A and the no-cycle condition. We stated the above fact.

Theorem 2.2 For $C^{1}$-generic $f$, if $f$ is expansive then $f$ satisfies both Axiom $\mathrm{A}$ and the no-cycle condition.

In this spirit, we show that $C^{1}$-generically, every continuum-wise expansive diffeomorphism satisfies both Axiom A and the no-cycle condition. This is a generalization of the remarkable result in [7].

Theorem A For $C^{1}$-generic $f$, if $f$ is continuum-wise expansive then $f$ satisfies both $A x$ iom $\mathrm{A}$ and the no-cycle condition.

\section{Continuum-wise volume-preserving diffeomorphisms}

Let $M$ be a closed $C^{\infty}$ Riemannian manifold endowed with a volume form $\omega$. Let $\mu$ denote the Lebesgue measure associated to $\omega$, and let $d$ denote the metric induced on $M$ by the Riemannian structure. Denote by $\operatorname{Diff}_{\mu}(M)$ the set of diffeomorphisms which preserves the Lebesgue measure $\mu$ endowed with the Whitney $C^{1}$-topology. Note that in volumepreserving diffeomorphisms, the non-wandering set $\Omega(f)=M$ by recurrent theorem. We say that $\Lambda$ is hyperbolic if the tangent bundle $T_{\Lambda} M$ has a $D f$-invariant splitting $E^{s} \oplus E^{u}$ and there exist constants $C>0$ and $0<\lambda<1$ such that

$$
\left\|\left.D_{x} f^{n}\right|_{E_{x}^{s}} ^{s}\right\| \leq C \lambda^{n} \quad \text { and } \quad\left\|\left.D_{x} f^{-n}\right|_{E_{x}^{u}}\right\| \leq C \lambda^{n}
$$


for all $x \in \Lambda$ and $n \geq 0$. Moreover, if $\Lambda=M$ then $f$ is Anosov. Note that $f$ is Anosov then $f$ is expansive, and so, $f$ is continuum-wise expansive. In [8], Bessa et al. proved that a volumepreserving diffeomorphism belongs to the $C^{1}$-interior of the set of all expansive volumepreserving diffeomorphisms if and only if it is Anosov. For the another conservative cases, that is, geodesic flow and a Hamiltonian system, Bessa et al. have shown in [9] that if a Hamiltonian system belongs to the $C^{2}$-interior of the set of all expansive Hamiltonian systems then it is Anosov. And Ruggiero [10] showed that if a geodesic flow belongs to the $C^{1}$-interior of the set of all expansive geodesic vector fields then it is Anosov.

Let $\mathcal{C} \mathcal{E}_{\mu}(M)$ be the set of all continuum-wise expansive volume-preserving diffeomorphisms. In this paper, we study the continuum-wise expansive case, and if $f$ belongs to the $C^{1}$-interior of $\mathcal{C} \mathcal{W} \mathcal{E}_{\mu}(M)$, then $f$ is Anosov. Let int $\mathcal{C} \mathcal{W} \mathcal{E}_{\mu}(M)$ denote the $C^{1}$-interior of the set of all continuum-wise expansive volume preserving diffeomorphisms. In this paper, we prove the following theorem.

Theorem B The set $\mathcal{A N}_{\mu}(M)$ of Anosov diffeomorphisms in $\operatorname{Diff}_{\mu}(M)$ coincides with the $C^{1}$-interior of the set of continuum-wise expansive diffeomorphisms in $\operatorname{Diff}_{\mu}(M)$; that is, $\mathcal{A N}_{\mu}(M)=\operatorname{int} \mathcal{C} \mathcal{W} \mathcal{E}_{\mu}(M)$

In diffeomorphisms, Arbieto [7] proved that $C^{1}$-generically, if $f$ is expansive then $f$ is $\Omega$-stable. It is well known that for a $\Omega$-stable diffeomorphism, there is a diffeomorphism such that the diffeomorphism is not expansive. However, for volume-preserving diffeomorphisms, the phenomenon cannot happen since $\Omega(f)=M$. $\operatorname{In} \operatorname{dim} M=2$, for $C^{1}$-generic $f$, if a $C^{1}$-neighborhood $\mathcal{U}(f)$ of $f$, there is $g \in \mathcal{U}(f)$ such that $g$ has a periodic point $p_{g}$ with homoclinic tangency $q_{g}$ then $f$ has a periodic point $p$ with homoclinic tangency $q$. In fact, it is closely related to the conjecture of Smale (see [11]). Note that if $\operatorname{dim} M=2$ then it does not exist normally hyperbolic. In this paper, we consider $\operatorname{dim} M \geq 3$. Recently, Bessa et al. [8] proved that $\operatorname{dim} M \geq 3$, for $C^{1}$-generic $f$, if $f \in \operatorname{Diff}_{\mu}(M)$ is expansive then $f$ is Anosov. For a Hamiltonian system, Lee [12] showed that $C^{2}$-generically, an expansive Hamiltonian system is Anosov. In this spirit, we study the continuum-wise expansiveness for generic view point. Then we have the following.

Theorem C For $C^{1}$-generic $f$, iff is continuum-wise expansive then it is transitive Anosov.

\section{Proof of Theorem A}

Let $\operatorname{dim} M \geq 3$ and let $f \in \operatorname{Diff}(M)$. We prepare several lemmas to arrive at Theorem A. The Franks lemma [13] will play an essential role in our proofs.

Lemma 4.1 Let $\mathcal{U}(f)$ be any given $C^{1}$-neighborhood of $f$. Then there exist $\varepsilon>0$ and a $C^{1}$ neighborhood $\mathcal{U}_{0}(f) \subset \mathcal{U}(f)$ of $f$ such that for given $g \in \mathcal{U}_{0}(f)$, a finite set $\left\{x_{1}, x_{2}, \ldots, x_{N}\right\}$, a neighborhood $U$ of $\left\{x_{1}, x_{2}, \ldots, x_{N}\right\}$ and linear maps $L_{i}: T_{x_{i}} M \rightarrow T_{g\left(x_{i}\right)} M$ satisfying $\| L_{i}-$ $D_{x_{i}} g \| \leq \varepsilon$ for all $1 \leq i \leq N$, there exists $\hat{g} \in \mathcal{U}(f)$ such that $\hat{g}(x)=g(x)$ if $x \in\left\{x_{1}, x_{2}, \ldots, x_{N}\right\} \cup$ $(M \backslash U)$ and $D_{x_{i}} \hat{g}=L_{i}$ for all $1 \leq i \leq N$.

Let $p$ be a periodic point of $f$, and let $0<\delta<1$. We say $p$ has a $\delta$-weak eigenvalue if $D_{p} f^{\pi(p)}$ has an eigenvalue $\lambda$ such that $(1-\delta)^{\pi(p)}<|\lambda|<(1+\delta)^{\pi(p)}$. The following lemma will also play a crucial role in our proof. 
Lemma 4.2 [7, Lemma 5.1] There exists a residual set $\mathcal{R}_{1} \subset \operatorname{Diff}(M)$ such that for any $f \in \mathcal{R}_{1}$,

(1) for any $\delta>0$, if for any $C^{1}$-neighborhood $\mathcal{U}(f)$, there is $g \in \mathcal{U}(f)$ which has a hyperbolic $p_{g} \in P(g)$ with a $\delta$-weak eigenvalue, then $f$ has a hyperbolic point $p \in P(f)$ with a $2 \delta$-weak eigenvalue;

(2) for any $\delta>0$, if $f$ has a hyperbolic point $q \in P(f)$ with a $\delta$-weak eigenvalue, then $f$ has a hyperbolic point $p \in P(f)$ with a $\delta$-weak eigenvalue, whose eigenvalues are all real.

Remark 4.3 If $f$ has a normally hyperbolic, then by Hirsh et al. [14] and Mañé [15], it is $C^{1}$-robust, that is, for any $g C^{1}$-close to $f, g$ has a normally hyperbolic then $f$ also has a normally hyperbolic (see also [16]).

Lemma 4.4 There exists a residual set $\mathcal{R}_{2} \subset \operatorname{Diff}(M)$ such that for $f \in \mathcal{R}_{2}$ if $f$ is continuum-wise expansive, then there exists $\delta>0$ such that $f$ has no $\delta$-weak eigenvalue.

Proof Let $\mathcal{R}_{2}=\mathcal{R}_{1}$, and let $f \in \mathcal{R}_{2}$ be continuum-wise expansive for $f$. Suppose, by contradiction, that for any $\delta>0$ there is a periodic point $p$ of $f$ such that $p$ has a $\delta$-weak eigenvalue. Let $\varepsilon>0$, and let $\mathcal{V}(f) \subset \mathcal{U}_{0}(f)$ be a $C^{1}$-neighborhood of $f$ which is given by Lemma 4.1 with respect to $\mathcal{U}_{0}(f)$. Then there exist $g \in \mathcal{U}(f)$ and a non-hyperbolic periodic point $q$ of $g$ such that an eigenvalue $\lambda$ of $D_{q} g^{\pi(q)}$ with $|\lambda|=1$, and $T_{q} M=E^{c}(q) \oplus E^{s}(q) \oplus$ $E^{u}(q)$, where $E^{\sigma}(q), \sigma=c, s, u$, are $D_{q} g^{\pi(q)}$-invariant subspaces corresponding to eigenvalues $\lambda$ of $D_{q} g^{\pi(q)}$ for $|\lambda|=1,|\lambda|<1$, and $|\lambda|>1$, respectively. Let $\mathcal{W}(f) \subset \mathcal{V}(f)$ be the $C^{1}$ $\varepsilon_{0}$-ball of $f$. Set $C=\sup _{x \in M}\left\{\left\|D_{x} g\right\|\right\}$. For $0<\varepsilon_{1}<\varepsilon_{0}$, we can obtain a linear automorphism $\mathcal{O}: T_{q} M \rightarrow T_{q} M$ such that

(i) $\|\mathcal{O}-\mathrm{id}\|<\frac{\varepsilon_{1}}{C}$,

(ii) $\mathcal{O}$ keeps $E^{\sigma}$ invariant, where $\sigma=c, s, u$,

(iii) all eigenvalues of $\mathcal{O} \circ D_{q} g^{\pi(q)}$, say $\mu_{j}, j=1,2, \ldots, c$, are roots of unity.

Let $F$ be the finite set $\left\{q, g(q), \ldots, g^{\pi(q)-1}(q)\right\}$. Define

$$
L_{j}= \begin{cases}D_{g^{j}(q)} g, & j=0,1, \ldots, \pi(q)-2, \\ \mathcal{O} \circ D_{g^{\pi(q)-1}(q)} g, & j=\pi(q)-1 .\end{cases}
$$

Observe that $\left\|L_{\pi(q)-1}-D_{g^{\pi(q)-1}(q) g} g \leq\right\| \mathcal{O}-\mathrm{id}\|\cdot\| D_{g^{k-1}(q)} g \|<\varepsilon_{0}$. Thus $\left\|L_{j}-D_{g^{j}(q)} g\right\|<\varepsilon_{0}$ for all $j=0,1, \ldots, \pi(p)-1$. By Lemma 4.1, we can find a diffeomorphism $g_{1} \in \mathcal{W}(f)$ and $\delta_{0}>0$ such that

(a) $B_{4 \delta_{0}}\left(g^{i}(q)\right) \cap B_{4 \delta_{0}}(q)=\emptyset, 0 \leq i \neq j \leq \pi(q)-1$,

(b) $g_{1}=g$ on $F \cup\left(M-\bigcup_{j=0}^{\pi(q)-1} B_{4 \delta_{0}}\left(g^{j}(q)\right)\right)$,

(c) $g_{1}=\exp _{g^{j+1}(q)} \circ L_{j} \circ \exp _{g^{j}(q)}^{-1}$ on $B_{\delta_{0}}\left(g^{j}(q)\right), 0 \leq j \leq \pi(q)-1$.

Define

$$
L=\mathcal{O} \circ D_{q} g^{\pi(q)}=\prod_{j=0}^{\pi(q)-1} L_{j},
$$

where $B_{\delta}(p)$ denotes the $\delta$-neighborhood of $p$.

Then by (iii) we can find $m>0$ such that $\left.L^{m}\right|_{E^{c}(q)}=\left.\mathrm{id}\right|_{E^{c}(q)}$. Choose a small $\delta_{1}$ satisfying $0<4 \delta_{1}<\delta_{0}$ such that

$$
L^{m k}\left(T_{q} M\left(4 \delta_{1}\right)\right) \subset T_{q} M\left(\delta_{0}\right),
$$


where $T_{q} M\left(\delta_{1}\right)=\left\{v \in T_{q} M \mid\|v\| \leq \delta_{1}\right\}$. Then by (c) we have

$$
\left(g_{1}^{\pi(q)}\right)^{m}=g_{1}^{m \pi(q)}=\exp _{q} \circ G^{m} \circ \exp _{q}^{-1}
$$

on $\exp _{q}\left(T_{q} M\left(4 \delta_{1}\right)\right)$.

We write

$$
T_{q} M\left(\delta_{1}\right)=E^{c}\left(q, \delta_{1}\right) \oplus E^{s}\left(q, \delta_{1}\right) \oplus E^{u}\left(q, \delta_{1}\right),
$$

where $E^{\sigma}\left(q, \delta_{1}\right)=E^{\sigma}(q) \cap T_{q} M\left(\delta_{1}\right), \sigma=c, s, u$. Then $\exp _{q}\left(E^{c}\left(q, 4 \delta_{1}\right)\right)$ is $\left(g_{1}^{k}\right)^{m}$-invariant. Since $f \in \mathcal{R}_{1}$, we assume that the eigenvalue $\lambda \in \mathbb{R}$.

Put $\exp _{q}\left(E^{c}\left(q, 4 \delta_{1}\right)\right)$ is an $\operatorname{arc} \mathcal{I}_{q}$ centered at $q$. Observe that $\left(g_{1}^{k}\right)^{m}=$ id on $\exp _{q}\left(E^{c}\left(q, 4 \delta_{1}\right)\right)$. By our construction, $\left(g_{1}^{k}\right)^{m}$ is the identity on the $\operatorname{arc} \mathcal{I}_{q}$. It is clear that the small $\operatorname{arc} \mathcal{I}_{q}$ is normally hyperbolic for $g_{1}$. By Remark 4.3, for any $g C^{1}$-close to $f$, if $g$ has a normally hyperbolic then $f$ has a normally hyperbolic, that is, it is $C^{1}$-robust. Then we know that $f$ has a small arc $\mathcal{J}_{q}$ which centered at $q$ with $f^{\pi(q)}\left(\mathcal{J}_{q}\right)=\mathcal{J}_{q}$. Note that if $f$ is continuum-wise expansive then $f^{k}$ is continuum-wise expansive for any $k \in \mathbb{Z}$ (see [2, Proposition 2.6]). Denote by $l(A)$ the length of $A$. Take $e=2 l\left(\mathcal{J}_{q}\right)$. Since $\mathcal{J}_{q}$ is $f^{\pi(q)}$-invariant, for all $n \in \mathbb{Z}$,

$$
\operatorname{diam}\left(f^{n}\left(\mathcal{J}_{q}\right)\right)<e .
$$

This is a contradiction.

We say that $f$ satisfies star condition if there is a $C^{1}$-neighborhood $\mathcal{U}(f)$ such that for any $g \in \mathcal{U}(f)$, every $p \in P(g)$ is hyperbolic. We denote by $\mathcal{F}(M)$ the set of diffeomorphisms satisfying star condition.

Lemma 4.5 There is a residual set $\mathcal{R}_{3} \subset \operatorname{Diff}(M)$ such that for any continuum-wise expansive map $f \in \mathcal{R}_{3}, f \in \mathcal{F}(M)$.

Proof Let $\mathcal{R}_{3}=\mathcal{R}_{2}$, and let $f \in \mathcal{R}_{3}$ be continuum-wise expansive. Proof by contradiction, we may assume that $f \notin \mathcal{F}(M)$. Then by Lemma 5.1, there is $g C^{1}$-close to $f$ and $p_{g} \in P(g)$ such that for any $\delta>0, p_{g}$ has a $\delta / 2$-weak eigenvalue. By Lemma 4.2, $p \in P(f)$ has a $\delta$-weak eigenvalue. This is a contradiction by Lemma 4.4.

Proof of Theorem A Let $f \in \mathcal{R}_{3}$ be continuum-wise expansive. By Lemma 4.5, $f \in \mathcal{F}(M)$. Since $f \in \mathcal{F}(M)$, By Aoki [17] and Hayashi [18], we know that $f$ satisfies both Axiom A and the no-cycle condition. Thus it is $\Omega$-stable.

\section{Proof of Theorem B and Theorem C}

Let $M$ and let $f \in \operatorname{Diff}_{\mu}(M)$ be as before. To prove our result, we use the Franks lemma, which is proved in [19, Proposition 7.4].

Lemma 5.1 Let $f \in \operatorname{Diff}_{\mu}^{1}(M)$, and $\mathcal{U}$ be a $C^{1}$-neighborhood of $f$ in $\operatorname{Diff}_{\mu}^{1}(M)$. Then there exist a $C^{1}$-neighborhood $\mathcal{U}_{0} \subset \mathcal{U}$ off and $\varepsilon>0$ such that if $g \in \mathcal{U}_{0}$, any finite $f$-invariant set $E=\left\{x_{1}, \ldots, x_{m}\right\}$, any neighborhood $U$ of $E$ and any volume-preserving linear maps $L_{j}: T_{x_{j}} M \rightarrow T_{g\left(x_{j}\right)} M$ with $\left\|L_{j}-D_{x_{j}} g\right\| \leq \varepsilon$ for all $j=1, \ldots, m$, there is a conservative diffeomorphism $g_{1} \in \mathcal{U}$ coinciding with $f$ on $E$ and out of $U$, and $D_{x_{j}} g_{1}=L_{j}$ for all $j=1, \ldots, m$. 
We denote by $\mathcal{F}_{\mu}(M)$ the set of diffeomorphisms $f \in \operatorname{Diff}_{\mu}(M)$ which have a $C^{1}$ neighborhood $\mathcal{U}(f) \subset \operatorname{Diff}_{\mu}(M)$ such that for any $g \in \mathcal{U}(f)$, every periodic point of $g$ is hyperbolic.

Very recently, Arbieto and Catalan [20] proved that every volume-preserving diffeomorphism in $\mathcal{F}_{\mu}(M)$ is Anosov.

Theorem 5.2 [20, Theorem 1.1] Every diffeomorphism in $\mathcal{F}_{\mu}(M)$ is Anosov.

To prove Theorem B, it is enough to show that a continuum-wise expansive volumepreserving diffeomorphism $f \in \mathcal{F}_{\mu}(M)$.

Remark 5.3 Let $f \in \operatorname{Diff}_{\mu}^{1}(M)$. From the Moser theorem (see [21]), we can find a smooth conservative change of coordinates $\varphi_{x}: U(x) \rightarrow T_{x} M$ such that $\varphi_{x}(x)=0$, where $U(x)$ is a small neighborhood of $x \in M$.

Lemma 5.4 Iff $\in \operatorname{int} \mathcal{C} \mathcal{W} \mathcal{E}_{\mu}(M)$, then $f \in \mathcal{F}_{\mu}(M)$.

Proof Take $f \in \operatorname{int} \mathcal{C W E}_{\mu}(M)$, and $\mathcal{U}(f)$ a $C^{1}$-neighborhood of $f$. Let $\varepsilon>0$ and $\mathcal{V}(f) \subset$ $\mathcal{U}(f)$ be corresponding number and $C^{1}$-neighborhood given by Lemma 5.1. To derive a contradiction, suppose that there is a non-hyperbolic periodic point $p \in P(g)$ for some $g \in \mathcal{V}(f)$. To simplify the notation in the proof, we may assume that $g(p)=p$. Then there is at least one eigenvalue $\lambda$ of $D_{p} g$ such that $|\lambda|=1$. By making use of Lemma 5.1, we linearize $f$ at $p$ with respect to Moser's theorem, that is, by choosing $\alpha>0$ sufficiently small we construct $g_{1} C^{1}$-nearby $g$ such that

$$
g_{1}(x)= \begin{cases}\varphi_{p}^{-1} \circ D_{p} g \circ \varphi_{p}(x) & \text { if } x \in B_{\alpha}(p), \\ g(x) & \text { if } x \notin B_{4 \alpha}(p) .\end{cases}
$$

Then $g(p)=g_{1}(p)=p$. Thus $T_{p} M=E^{c} \oplus E^{\sigma}$, where $E_{p}^{c}$ associated to $\lambda=1$ and $E_{p}^{\sigma}$ associated to eigenvalues less than one and greater than one. Take $\eta=\alpha / 4$. Then we define $E^{c}(\eta) \cap$ $\varphi_{p}\left(B_{\alpha}(p)\right)=E^{c}(\eta)$.

Case 1. $\operatorname{dim} E_{p}^{c}=1$.

Since $p$ is non-hyperbolic for $g_{1}$, by our construction, we may assume that there is $l>0$ such that $D_{p} g_{1}^{l}(v)=v$ for any $v \in E_{p}^{c}(\eta) \cap \varphi_{p}\left(B_{\alpha}(p)\right)$. Take $v \in E_{p}^{c}(\eta)$ such that $\|v\|=\eta / 4$. Then we can find a small arc $\mathcal{I}_{p}=\varphi_{p}^{-1}(\{t v: 1 \leq t \leq \eta / 4\}) \subset B_{\alpha}(p)$ such that

(i) $g_{1}^{i}\left(\mathcal{I}_{p}\right) \cap g_{1}^{j}\left(\mathcal{I}_{p}\right)=\emptyset$ if $0 \leq i \neq j \leq l-1$,

(ii) $g_{1}^{l}\left(\mathcal{I}_{p}\right)=\mathcal{I}_{p}$, that is, $\left.g_{1}^{l}\right|_{\mathcal{I}_{p}}$ is the identity map,

(iii) $\mathcal{I}_{p}$ is normally hyperbolic.

For simplicity, we assume that $g_{1}^{l}=g_{1}$. Take $e=\eta$. Then for all $n \in \mathbb{Z}$,

$$
\operatorname{diam}\left(g_{1}^{n}\left(\mathcal{I}_{p}\right)\right)<e .
$$

This is a contradiction.

Case 2. $\operatorname{dim} E_{p}^{c}=2$.

In the proof of the second case, to avoid notational complexity, we consider the case $g(p)=p$. By Lemma 5.1, there is $\alpha>0$ and $h \in \mathcal{U}(f)$ such that $h(p)=g(p)=p$ and $h(x)=$ $\varphi_{p}^{-1} \circ D_{p} g \circ \varphi_{p}(x)$ if $x \in B_{\alpha}(p)$. With a small modification of $D_{p} g$, we may assume that there 
is $l>0$ such that $D_{p} g^{l}(v)=v$ for any $v \in E_{p}^{c}(\alpha)$ by Lemma 5.1. We can choose $v \in E_{p}^{c}(\alpha)$ such that $\|v\|=\alpha / 4$ and we set $\mathcal{D}_{p}=\varphi_{p}^{-1}(\{t v: 1 \leq t \leq \alpha / 4\}) \subset B_{\alpha}(p)$. Then the disk $\mathcal{D}_{p}$ satisfies the following conditions:

(i) $h^{i}\left(\mathcal{D}_{p_{h}}\right) \cap h^{j}\left(\mathcal{D}_{p_{h}}\right)=\emptyset$ if $0 \leq i \neq j \leq l-1$,

(ii) $h^{l}\left(\mathcal{D}_{p_{h}}\right)=\mathcal{D}_{p_{h}}$, that is, $\left.h^{l}\right|_{\mathcal{D}_{p_{h}}}$ is the identity map,

(iii) $\mathcal{D}_{p}$ is normally hyperbolic.

As in the proof of the $\operatorname{dim} E_{p}^{c}=1$, we can derive a contradiction.

Proof of Theorem B Suppose that $f \in \operatorname{int} \mathcal{C} \mathcal{W} \mathcal{E}_{\mu}(M)$. By Lemma 5.4, $f \in \mathcal{F}_{\mu}(M)$. Thus by Theorem 5.2, $f$ is Anosov.

Proof of Theorem C The proof of Theorem C is parallel the proof of Theorem A. Indeed, to prove Theorem A we use previous results - Lemmas 4.2, 4.4 and 4.5. Then we have a volume-preserving diffeomorphism $f \in \mathcal{F}_{\mu}(M)$. Thus $f$ is Anosov.

In diffeomorphisms, there is an open problem: are Anosov diffeomorphisms transitive? In [13] Franks and [22] Newhouse proved it for codimension one Anosov diffeomorphisms. It was announced in Xia in a talk, Anosov diffeomorphisms are transitive, an invited talk of the Rocky Mountain Conference on Dynamical Systems, May 12-14, 2008 , that every Anosov diffeomorphism is transitive. It has not been published yet. Nevertheless, in the volume-preserving diffeomorphism, an Anosov diffeomorphism has the non-wandering set equal to the whole manifold $M$ by the Poincare theorem. By the shadowing property of the hyperbolic sets the periodic points are dense in $M$. And by the Smale spectral decomposition theorem, we have a single piece equal to $M$, and so, we have transitivity. Thus, the Anosov volume-preserving diffeomorphism is transitive, which is a direct consequence of classic hyperbolic dynamics. But in volume-preserving diffeomorphisms Bonatti and Crovisier proved that $C^{1}$-generically, a volume-preserving diffeomorphism is transitive.

Theorem 5.5 [23, Theorem 1.3] There is a residual set $\mathcal{R}_{4} \subset \operatorname{Diff}_{\mu}(M)$ such that for any $f \in \mathcal{R}_{4}, f$ is transitive and $M$ is a unique homoclinic class.

We say that $f$ is transitive if there is a point $x \in M$ such that $\omega(x)=M$, where $\omega(x)$ is the omega limit set.

Remark 5.6 In [24, Theorem 1.3], Newhouse showed that $C^{1}$-generic volume-preserving diffeomorphisms in surfaces are Anosov or else the elliptical points, nonreal eigenvalues conjugated and of norm one, are dense.

By [8] and Theorem A, we have the following.

Corollary 5.7 There is a residual set $\mathcal{G} \subset \operatorname{Diff}_{\mu}(M)$ such that for any $f \in \mathcal{G}$, the following are equivalents:

(a) $f$ is expansive,

(b) $f$ is transitive Anosov.

Moreover, if $\operatorname{dim} M \geq 3$ then

(c) $f$ is continuum-wise expansive, 
(d) $f$ has the shadowing property,

(e) $f$ has the weak specification property.

Proof Let $f \in \mathcal{G}=\mathcal{R}_{3} \cap \mathcal{R}_{4}$ is continuum-wise expansive. By Theorem A, $f$ is Anosov. Since $f \in \mathcal{R}_{4}$, by Lemma $5.5, f$ is transitive. Thus if $f$ is continuum-wise expansive, then $f$ is transitive Anosov. By Bessa et al. [8], $f$ is expansive, then $f$ is Anosov, and so, $f$ is transitive Anosov. If $\operatorname{dim} M \geq 3$, then by Bessa et al. [8] if $f$ has the shadowing property and $f$ has the weak specification property, then $f$ is Anosov and since $f \in \mathcal{R}_{4}$, also $f$ is transitive. Thus $f$ is transitive Anosov.

\section{Competing interests}

The author declares to have no competing interests.

\section{Acknowledgements}

The author would like to thank the referee for his/her useful comments, detailed corrections and suggestions. This work is supported by Basic Science Research Program through the National Research Foundation of Korea (NRF) funded by the Ministry of Science, ICT \& Future Planning (No. 2014R1A1A1A05002124).

Received: 9 May 2014 Accepted: 28 July 2014 Published: 01 Oct 2014

\section{References}

1. Mañé, R: Expansive diffeomorphisms. In: Dynamical Systems. Lecture Notes in Mathematics, vol. 468, pp. 162-174. Springer, Berlin (1975)

2. Kato, H: Continuum-wise expansive homeomorphisms. Can. J. Math. 45, 576-598 (1993)

3. Kato, H: Concerning continuum-wise fully expansive homeomorphisms of continua. Topol. Appl. 53, $239-258$ (1993)

4. Das, T, Lee, K, Lee, M: C $C^{1}$-Persistently continuum-wise expansive homoclinic classes and recurrent sets. Topol. Appl. 160, 350-359 (2013)

5. Sakai, K: Continuum-wise expansive diffeomorphisms. Publ. Mat. 41, 375-382 (1997)

6. Lee, M: Continuum-wise expansive and dominated splitting. Int. J. Math. Anal. 23, 1149-1154 (2013)

7. Arbieto, A: Periodic orbits and expansiveness. Math. Z. 269, 801-807 (2011)

8. Bessa, M, Lee, M, Wen, X: Shadowing, expansiveness and specification for $C^{1}$-conservative systems. arXiv:1305.3473v1

9. Bessa, M, Rocha, J, Torres, MJ: Shades of hyperbolicity for Hamiltonians. Nonlinearity 26, 2851-2873 (2013)

10. Ruggiero, RO: Persistently expansive geodesic flows. Commun. Math. Phys. 140, 203-215 (1991)

11. Bonatti, C, Díaz, LJ: Abundance of C C -robust homoclinic tangencies. Trans. Am. Math. Soc. 364, 5111-5148 (2012)

12. Lee, M: Generic expansive Hamiltonian systems. Chaos Solitons Fractals 61, 24-26 (2014)

13. Franks, J: Anosov diffeomorphisms. In: Global Analysis (Proc. Symp. Pure Math., Berkely, California, 1968), vol. XIV, pp. 61-93. Am. Math. Soc., Providence (1970)

14. Hirsh, M, Pugh, C, Shub, M: Invariant Manifolds. Lecture Notes in Mathematics. Springer, Berlin (1977)

15. Mañé, R: Persistent manifolds are normally hyperbolic. Trans. Am. Math. Soc. 246, 261-283 (1978)

16. Gan, S, Yang, D: Expansive homoclinic classes. Nonlinearity 22, 729-733 (2009)

17. Aoki, N: The set of Axiom A diffeomorphisms with no cycle. Bol. Soc. Bras. Mat. 23, 21-65 (1992)

18. Hayashi, S: Diffeomorphisms in $\mathcal{F}^{1}(M)$ satisfy Axiom A. Ergod. Theory Dyn. Syst. 12, 233-253 (1992)

19. Bonatti, C, Díaz, LJ, Pujals, ER: A C $C^{1}$-generic dichotomy for diffeomorphisms: weak forms of hyperbolicity or infinitely many sinks or sources. Ann. Math. 158, 355-418 (2003)

20. Arbieto, A, Catalan, T: Hyperbolicity in the volume preserving scenario. Ergod. Theory Dyn. Syst. 33, 1644-1666 (2013)

21. Moser, J: On the volume elements on a manifold. Trans. Am. Math. Soc. 120, 286-294 (1965)

22. Newhouse, S: On codimension one Anosov diffeomorphisms. Am. J. Math. 92, 761-770 (1970)

23. Bonatti, C, Crovisier, S: Récurrence et généricité. Invent. Math. 158, 33-104 (2004)

24. Newhouse, S: Quasi-elliptic periodic points in conservative dynamical systems. Am. J. Math. 99, 1061-1087 (1977)

10.1186/1029-242X-2014-379

Cite this article as: Lee: Continuum-wise expansive diffeomorphisms and conservative systems. Journal of Inequalities and Applications 2014, 2014:379 